\title{
Influenza D Virus: A Potential Threat for Humans? ${ }^{\dagger}$
}

\author{
Claudia Maria Trombetta 1,*, Serena Marchi ${ }^{1}$, Ilaria Manini ${ }^{1}$, Otfried Kistner ${ }^{2}$, Feng Li ${ }^{3}$, \\ Pietro Piu 2, Alessandro Manenti ${ }^{4}$, Fabrizio Biuso 4, Chithra Sreenivasan ${ }^{3}$, Julian Druce 5 \\ and Emanuele Montomoli 1,2,4 \\ 1 Department of Molecular and Developmental Medicine, University of Siena, Via Aldo Moro, \\ 53100 Siena, Italy; serena.marchi2@unisi.it (S.M.); ilaria.manini@unisi.it (I.M.); \\ emanuele.montomoli@unisi.it (E.M.) \\ 2 VisMederi srl, Strada del Petriccio e Belriguardo 35, 53100 Siena, Italy; kistner@vismederi.com (O.K.); \\ piu@vismederi.com (P.P.) \\ 3 Department of Biology and Microbiology, South Dakota State University, Brookings, SD 57007, USA; \\ feng.li@sdstate.edu (F.L.); Chithra.Sreenivasan@sdstate.edu (C.S.) \\ 4 VisMederi Research srl, Strada del Petriccio e Belriguardo 35, 53100 Siena, Italy; \\ alessandro.manenti@vismederiresearch.com (A.M.); biuslee@yahoo.it (F.B.) \\ 5 Victorian Infectious Diseases Reference Laboratory, 792 Elizabeth Street, Melbourne, VIC 3000, Australia; \\ julian.druce@mh.org.au \\ * Correspondence: trombetta@unisi.it \\ † Presented at Viruses 2020-Novel Concepts in Virology, Barcelona, Spain, 5-7 February 2020. \\ Published: 11 June 2020
}

\begin{abstract}
Influenza D virus (IDV) is a novel influenza virus first isolated from swine in 2011 in Oklahoma. Several studies have isolated IDV in cattle from multiple geographic areas, suggesting that cattle could be a possible primary natural reservoir for the virus. To date, few studies have been performed on human samples and there is no conclusive evidence that IDV has the ability to infect humans. This serological study aimed to assess the prevalence of antibodies against IDV in the human population. The IDV used in the serological analysis was influenza D/bovine/Oklahoma/660/2013. The human serum samples, collected in Italy between 2005 and 2017, were randomly selected from the laboratory serum bank and tested by the haemagglutination inhibition (HI) assay. HI positivity has been confirmed using the virus neutralization (VN) assay. Based on HI positivity (HI titers $\geq 10$ ), a low prevalence (5\%-10\%) was observed between 2005 and 2007. There has been a sharp increase since 2008, resulting in two main peaks in 2009-2010 and 2013-2014, a finding confirmed by the statistical trend analysis. The same pattern and trends can be seen with higher HI titers of $>20$ and $\geq 40$. The prevalence of antibodies against IDV has increased in the human population in Italy from 2005 to 2017. Low prevalence values between 2005 and 2007 suggest that IDV most probably circulated before its detection in 2011, and perhaps even before 2005. In Italy, IDV has been shown to circulate among swine and bovine herds. It is, therefore, possible that prevalence peaks in humans follow the infection epidemics in animals and do not to persist in the population, resembling a spillover event from the animal reservoir and showing that the virus may not circulate consistently in the human population. However, IDV seemed to have the ability to elicit an immune response in humans.
\end{abstract}

Keywords: influenza D virus; seroprevalence; Italy; humans

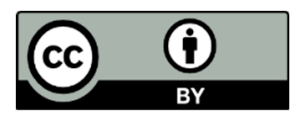

(C) 2020 by the authors. Licensee MDPI, Basel, Switzerland. This article is an open access article distributed under the terms and conditions of the Creative Commons Attribution (CC BY) license (http://creativecommons.org/licenses/by/4.0/). 